\title{
Advancing Health Systems and Pharmaceutical Relations: Best Practices for Population Health
}

\author{
Richard G. Stefanacci, DO, MGH, MBA, AGSF, CMD ${ }^{1,2}$ and Scott Guerin, $\mathrm{PhD}^{1}$
}

$\mathbf{H}$ EALTH SYSTEMS CONTINUE to become bigger, gaining market clout as evidenced by PricewaterhouseCooper proclaiming 2016 "the year of merger mania.," 1 As health systems expand, a corresponding trend has emerged to increase the level of risk and clinical integration, with a shift in focus to efficient and effective population health management.

This shift in focus was demonstrated in a national survey we conducted with 27 executives from a wide range of health systems in terms of location, size, and level of integration. Perhaps the most telling response was related to the level of clinical integration in their organizations now versus 3,5 , and 10 years in the future on a scale of 0 (no integration/risk) to 5 (full risk integrated). On average, the health systems indicated they were currently at a level of 2.5 , but would steadily increase to a full risk integrated model similar to Kaiser Health System over the next decade (unpublished data; The Access Group Integrated Health System Survey; September 26, 2016).

Success for full-risk integrated health systems relies on thoughtful management of all aspects of care including pharmaceutical treatments, and on this point, health system respondents expressed a strong interest in controlling outpatient treatment selections across several patient populations over the next several years (Fig. 1). Importantly, a follow-up question indicated that the organizations are very confident that they will gain this control. Most likely, the driver of this change is the anticipation of accepting a higher level of risk, with outcomes tied to improving clinical and financial outcomes.

Part of health systems' management of pharmaceuticals can come from their development and enforcement of preferred outpatient drug lists. The executives participating in this survey agreed that this will be a strong trend over the next 3 to 5 years and project that the use of outpatient drug lists will almost double during this time frame. Leading factors in determining an outpatient preferred drug list center on delivery of accountable outcomes, overall reduction in total cost of care, and improved efficacy over competitor treatments. The impact on patient quality of life was rated last on the list of factors.

\section{Health System Management of Pharmaceuticals}

Health systems evaluate and manage pharmaceuticals differently than traditional managed care organizations (MCO). MCOs are focused on contracting for price, whereas health systems can create contracts based on unique service offerings, especially given that many are not yet payers so contracting for price would not be possible. In addition, treatment decisions in MCOs are typically made by pharmacy directors with annual contracts compared with the multiyear contracts made by chief medical officers in health systems. The 2 models also differ in their ability to influence provider and patient behavior. MCOs can set formularies and treatment protocols offering financial incentives for provider compliance. Patients can be encouraged by their physicians to adhere to their treatment plan by engaging them in the decisionmaking process. But health systems have more control levers at their disposal. For example, health systems formularies and treatment guidelines can be embedded in a system's electronic medical records that are set to guide clinical decisions to specific protocols and treatment options. Also, health system patient portals can be used to direct patients to the clinical options the health system has adopted in addition to offering patient education, adherence programs, and communication options; these portals tend to be more trusted than those of MCOs.

\section{Model for Successful Health System Pharmaceutical Partnership}

Traditionally, pharmaceutical manufacturers have concentrated on gaining access through payers via their marketing and educational efforts focused solely on their product. In contrast, the health system has a much broader view than a single treatment, which places emphasis on prevention and addressing changes in lifestyle that help limit diseases from progressing to the point that requires clinical intervention. This focus comes from the health system's greater emphasis on population health management and provider integration than a traditional payer. The population health perspective emphasizes the idea that if more resources are deployed "upstream" to increase disease awareness,

${ }^{1}$ Government Policy Systems and Analytics, The Access Group, Berkeley Heights, New Jersey.

${ }^{2}$ College of Population Health, Thomas Jefferson University, Philadelphia, Pennsylvania.

(C) Richard G. Stefanacci and Scott Guerin 2018; Published by Mary Ann Liebert, Inc. This article is available under the Creative Commons License CC-BY-NC (http://creativecommons.org/licenses/by-nc/4.0). This license permits non-commercial use, distribution and reproduction in any medium, provided the original work is properly cited. Permission only needs to be obtained for commercial use and can be done via RightsLink. 


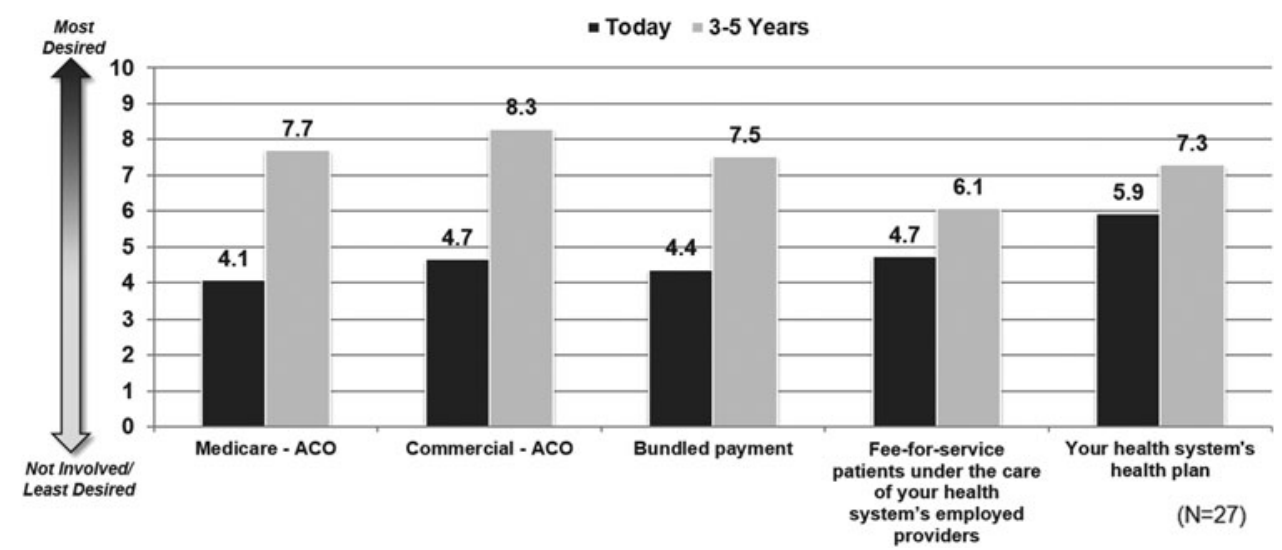

FIG. 1. Desire to control outpatient treatment selections. ACO, accountable care organization. Source: Data on file. Integrated Health System Survey. September 26, 2016. The Access Group.

modify lifestyle behavior, and increase preventive action, the cost savings will be substantial because the "downstream" medical costs will be minimized or avoided.

Pharmaceutical manufacturers that understand this broader perspective and the specific needs of health systems can be viewed as a true partner providing a service solution, rather than simply a drug sales organization selling a product. A few manufacturers are seeing this trend and responding by establishing field teams and internal departments that specifically target health systems with tools and resources to support population health. These resources include disease-specific patient education, screening and prevention materials, decision-support tools, and field training to educate the account teams on how to speak to health systems in terms that are important to them. This approach of engaging a health system begins with the manufacturer first articulating value by demonstrating how its treatment aligns with the goals of the health system. This would include the clinical background information typically found in product background presentations, in addition to any health economic and outcomes research data. The population health materials and resources can then be introduced to support the health system's broader focus in managing the disease. One difficulty for manufacturers when approaching health systems about population health resources is that initial materials should be above brand, avoiding branded product information. Specific product mentions can raise issues around the legitimacy of the tools and materials, possibly skewing the information and steering people to a specific product, rather than providing population health solutions.

An additional manufacturer's approach to health systems can come from providing tools to help change the behavior of both providers and patients. The product value story is an essential first step to provide a reason to make a change; however, as already mentioned, health systems have the advantage of being able to format their electronic medical record systems and patient portals. For providers, these systems can contain features to enforce adherence to a preferred formulary, flag missed appointments or refills, and present clinical data that indicate clinical action. Patient portals can serve as appointment reminders and contain adherence tools, along with diseaseand treatment-specific information.

As health systems continue to expand and evolve to address the health of populations, the way pharmaceutical manufacturers interact with them is changing as well, with both groups moving from transactional fee for service to value-based offerings. Manufacturers are beginning to adapt to this new environment by becoming a true partner to health systems through providing resources that align with the system's clinical and business priorities. Most importantly, manufacturers are providing effective services to help change provider and patient behavior to ultimately improve the health of populations.

\section{Author Disclosure Statement}

The authors are employees of The Access Group that funded the survey.

\section{Reference}

1. PwC. Merger Mania, Drug Pricing and New Tech: PwC's Health Research Institute List of Top Health Industry Trends for 2016 (News Release). December 9, 2015. www.pwc .com/us/en/press-releases/2015/pwc-top-health-issues-2016press-release.html Accessed January 25, 2017.

Address correspondence to: Scott Guerin, PhD

Government Policy Systems and Analytics The Access Group 400 Connell Drive 2nd Floor

Berkeley Heights, NJ 07922

E-mail: sguerin@theaccessgp.com 